\title{
The Galactic center: a model for cosmic ray interactions in starburst galaxies?
}

\author{
T. Yoast-Hull ${ }^{1,2}$, J. S. Gallagher III $^{3}$ and E. Zweibel ${ }^{1,2,3}$ \\ ${ }^{1}$ Department of Physics, University of Wisconsin-Madison, Madison, WI 53706, USA \\ email: yoasthull@wisc.edu \\ ${ }^{2}$ Center for Magnetic Self-Organization in Laboratory and Astrophysical Plasmas, University \\ of Wisconsin-Madison, WI, USA \\ ${ }^{3}$ Department of Astronomy, University of Wisconsin-Madison, Madison, WI 53706, USA
}

\begin{abstract}
The Galactic center contains strong magnetic fields, high radiation fields, and dense molecular gas, as is also the case in starburst galaxies. The close proximity of the Galactic center allows for more and better observations of the interstellar medium than for extragalactic sources making it an ideal place for testing models for cosmic ray interactions. We compare our semianalytic model for cosmic ray interactions to published data for both the Galactic center and the starburst galaxy NGC 253. We present the predicted radio and $\gamma$-ray spectra and compare the results with published measurements. In this way we provide a quantitative basis for assessing the degree to which the Galactic center resembles a starburst system.
\end{abstract}

Keywords. cosmic rays, Galaxy: center - galaxies: individual (NGC253) - galaxies: starburst - gamma rays: galaxies — radio continuum: galaxies

\section{Introduction}

Previously, we developed a single-zone model for cosmic ray interactions in starburst nuclei (Yoast-Hull et al. 2013a). Due to the observed similarities between the Galactic center and starburst nuclei, we now compare the model to observations of the Galactic center and of the starburst galaxy NGC 253.

In the model, we assume that cosmic rays sample the mean density of a three-phase interstellar medium (ISM) (Boettcher et al. 2013). Due to the extremely high densities in starburst nuclei, energy losses dominate and so we neglect cosmic ray diffusion and only consider energy and advective losses, applying the model to the central molecular zone (CMZ). Additionally, we assume a constant particle acceleration efficiency from supernovae with a power-law injection spectrum. From a primary cosmic ray population, we calculate a secondary population of cosmic ray electrons and positrons. Both the primary and secondary populations of electrons/positrons are included in our models for the radio spectrum.

\section{Results}

We use a $\chi^{2}$ test to compare our model to both $\gamma$-ray and radio data. For the $\gamma$-ray spectrum, the model includes neutral pion decay, bremsstrahlung, and inverse Compton emission. The radio spectrum is fit with both non-thermal synchrotron emission in the hot, diffuse medium and free-free absorption and emission from warm, ionized gas clouds. The goodness of the fit depends on molecular gas mass, ionized gas density, magnetic field strength, advection speed, and absorption fraction. We find that blindly applying 


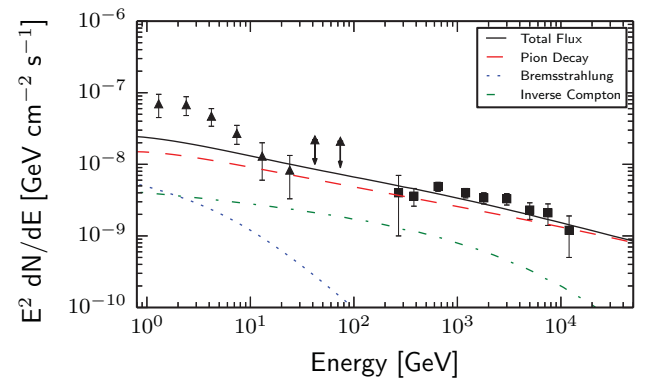

(a) GC $\gamma$-Ray Flux

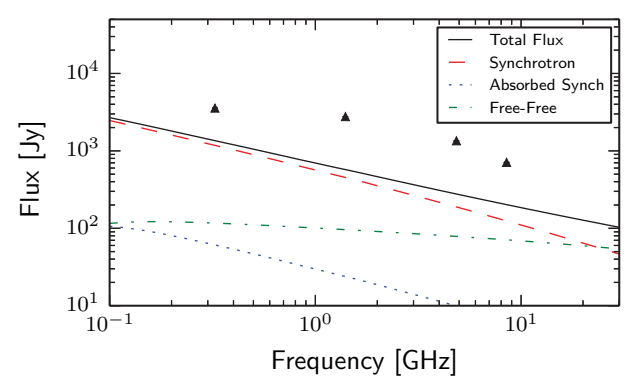

(c) GC Radio Spectrum

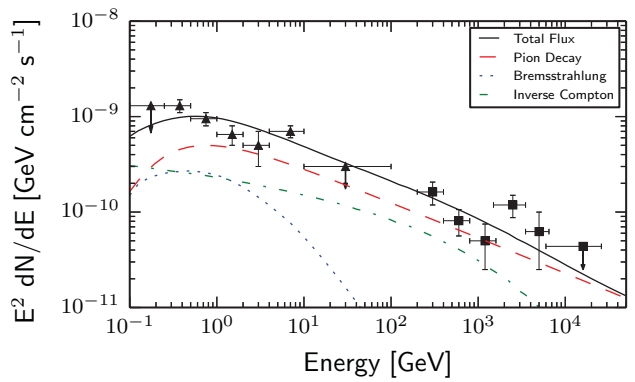

(b) NGC $253 \gamma$-Ray Flux

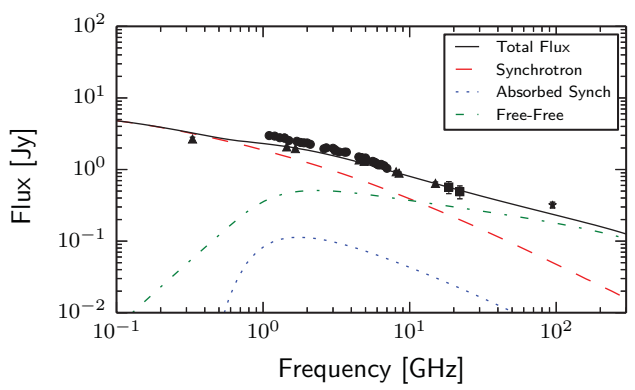

(d) NGC 253 Radio Spectrum

Figure 1. Data from Yusef-Zadeh et al. (2013), Abramowski et al. (2012), Carilli (1996), Paglione \& Abrahams (2012), Peng et al. (1996), Ricci et al. (2006), Williams \& Bower (2010). [A COLOR VERsion is aVAILAble online.]

the model to the Galactic center results in a $\gamma$-ray spectrum which does not fit the observed Fermi data.

\section{Conclusions}

Although the model works well for NGC 253 (Yoast-Hull et al. 2014), from the excess of hadronic emission at $\mathrm{TeV}$ energies and lack of leptonic emission at $\mathrm{GeV}$ energies, it is clear that the starburst model fails for the Galactic center. The underestimated $\gamma$-ray and radio spectra suggest that the electron to proton ratio is too low by a factor of $\sim 8$. An excess of free-free emission in the radio spectrum is another difficulty for this model.

\section{Acknowledgements}

This work was supported in part by NSF AST-0907837, NSF PHY-0821899 (to CMSO), and NSF PHY-0969061 (to the IceCube Collaboration).

\section{References}

Abramowski, A., Acero, F., Aharonian, F., et al. 2012, ApJ 757, 158

Boettcher, E., Zweibel, E. G., Yoast-Hull, T. M., \& Gallagher, III, J. S. 2013, ApJ in press Carilli, C. L. 1996, A\&A 305, 402

Paglione, T. A. D.. \& Abrahams, R. D. 2012, ApJ 755, 106

Peng, R., Zhou, S., Whiteoak, J. B., Lo, K. Y., \& Sutton, E. C. 1996, ApJ 470, 821 
Ricci, R., Prandoni, I., Gruppioni, C., Sault, R. J., \& de Zotti, G. 2006, A\&SA 445, 465 Williams, P. K. G.. \& Bower, G. C. 2010, ApJ 710, 1462

Yoast-Hull, T. M., Everett, J. E., Gallagher, III, J. S., \& Zweibel, E. G. 2013a, ApJ 768, 53

Yoast-Hull, T. M., Gallagher, III, J. S., Zweibel, E. G., \& Everett, J. E. 2014, ApJ 780, 137

Yusef-Zadeh, F., Hewitt, J. W., Wardle, M., et al. 2013, ApJ 762, 33 